\title{
Blob sizes and velocities in the Alcator C-Mod scrape- off layer
}

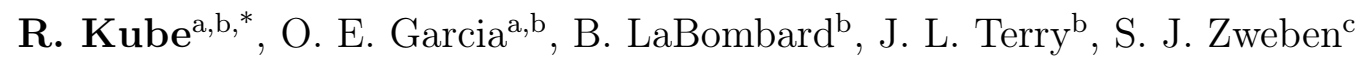

a Department of Physics and Technology, University of Tromsø, N-9037 Tromsø, Norway

b MIT Plasma Science and Fusion Center, Cambridge, MA, 02139, USA

c Princeton Plasma Physics Laboratory, Princeton, NJ, 08543, USA

PACS numbers: 52.30.-q 52.35.Ra 52.55.Dy 52.70.Kz

PSI-20 keywords: Alcator C-Mod, Cross-field transport, Fluctuations \& turbulence

\begin{abstract}
Gas-puff imaging data is used to investigate the dynamics of blob-like structures in the scrape-off layer of Alcator C-Mod. A blob-tracking algorithm is developed and used to compute blob propagation velocities and cross-field sizes for a series of discharges where the line-averaged particle density $\bar{n}_{\mathrm{e}}$ is varied. We find that the mean radial blob velocity increases from $150 \mathrm{~ms}^{-1}$ for $\bar{n}_{\mathrm{e}} / n_{G}=0.15$ to $450 \mathrm{~ms}^{-1}$ for $\bar{n}_{\mathrm{e}} / n_{G}=0.48$, where is the $n_{G}$ the Greenwald density. The cross-field size of the blobs is independent of the line-averaged particle densities. With increasing $\bar{n}_{\mathrm{e}} / n_{G}$ the observed blob velocities become larger than predicted by simple blob models for sheath-connected plasma filaments.
\end{abstract}

Corresponding author address: Department of Physics and Technology, University of Troms $\varnothing$, N-9037 Troms $\varnothing$, Norway

Corresponding author email: ralph.kube@uit.no 


\section{Introduction}

In the edge of toroidally magnetized plasmas, one universally observes intermittent, large density fluctuations, the source of which can be traced to large radial transport events. These transport events are believed to be associatde with cross-field motion of isolated plasma filaments that are aligned with the magnetic field and have significantly higher particle density and temperature than the ambient plasma. These coherent filaments are localized in the drift-plane perpendicular to the magnetic field, thus they are often referred to as blobs $[1,2,3]$.

A blob's particle density gradient perpendicular to the magnetic field gives rise to electric currents localized within the blob. Theoretical models use different closures for this electric current. Depending on the magnitude of individual current channels and the shape of the blob, one finds two velocity scaling regimes for blobs.

In one limit, a blob is assumed to have no structure along the magnetic field and to terminate at the sheaths that are formed where magnetic field lines intersect material walls. For large cross-field blob sizes $\ell$, electric currents in the drift-plane are balanced by sheath currents, and the radial blob velocity depends on the size as $V_{\text {rad }} \sim \ell^{-2}[1,2]$. This is often referred to as sheath-dissipative velocity scaling. For small cross-field size, the diamagnetic and polarization current balance and $V_{\text {rad }} \sim \ell^{1 / 2}$, which we call inertial blob velocity scaling $[1,2,4]$. Recent work revealed the velocity dependence of blobs on blob size and relative amplitude $\triangle n / N[5]$ :

$$
\frac{V_{\mathrm{rad}}}{V_{*}}=\frac{c_{2}}{2}\left(\frac{\ell}{\ell_{*}}\right)^{3}\left[-1+\left(1+\frac{c_{1}}{c_{2}^{2}} \frac{4 \ell_{*}^{5}}{\ell^{5}} \frac{\triangle n / N}{1+\triangle n / N}\right)^{1 / 2}\right] .
$$

Here $C_{s}$ is the ion acoustic velocity, $V_{*} / C_{s}=\left(8 \rho_{s}^{2} L_{\|} / R^{3}\right)^{1 / 5}$ and $\ell_{*}=\left(\rho_{s}^{4} L_{\|}^{2} / R\right)^{1 / 5}$ are derived from an order of magnitude estimate of the length scale at which all electric current channels balanced [1], $\rho_{s}$ is the thermal ion gyroradius, $L_{\|} / 2$ is the connection length and $R$ the curvature radius of the plasma. The coefficients $c_{1}$ and $c_{2}$ depend on the blob amplitude and have been obtained from numerical simulations [5]. From Eqn. (1) the inertial and sheath-dissipative velocity scaling are recovered in the limit $\ell \ll \ell_{*}$ and 
$\ell \gg \ell_{*}$ respectively. The size dependence of the blob velocity is in excellent agreement with recent measurements on the TORPEX device [6].

In another limit, the blob is assumed to be ballooned on the low-field side with a peak amplitude at the outboard-midplane. This strong modulation implies that parallel electric currents have little influence on filament dynamics [4]. In this limit, the inertial velocity scaling holds for all blob sizes and can be regarded as an upper limit for radial blob velocity.

The transition between blobs modulated at the low-field side and blobs perfectly elongated along the magnetic field lines is believed to be regulated by plasma collisionality $[7,8]$. Weak plasma collisionality permits large electric current along the magnetic field lines in the SOL. This impedes the radial velocity of a blob, giving it more time to expand along the field lines as it propagates through the SOL. With increasing collisionality, electric currents parallel to the magnetic field are impeded which results in faster radial blob propagation [9].

It has been shown, that plasma cross-field transport across the separatrix in Alcator CMod increases with line averaged density. This is believed to be due to increasing local collisionality [10]. In this contribution we explicitly study the effect of varying plasma line average density on blob dynamics in the scrape-off layer using the gas-puff imaging diagnostic.

\section{Method}

Gas puff imaging is an active diagnostic that uses a neutral gas puff, usually Helium or Deuterium, to enhance local emissions caused by collisions with the edge plasma [11, 12]. In Fig. 1 we present a schematic view of the GPI diagnostic installed at the outboard midplane in Alcator C-Mod. Light from atomic emission lines is captured by a $62 \times 62$ fiber bundle, passed through a line filter for the He I neutral atom emission line at $\lambda=587.6 \mathrm{~nm}$, and observed by a fast camera operating at a framerate of $396 \mathrm{kHz}$, a frame integration time of $2 \mu \mathrm{s}$, and a resolution of $64 \times 64 \mathrm{px}$. For $B_{T}=5.4 \mathrm{~T}$ and $I_{p}=1.0 \mathrm{MA}$ the $6 \mathrm{~cm} \times 6 \mathrm{~cm}$ field-of-view of the camera is perpendicular to the magnetic field. Due to varying plasma 
parameters and smearing due to the toroidal extend of the gas puff, the smallest structures resolvable by the camera is approximately $\sim 0.2 \mathrm{~cm} \times 0.2 \mathrm{~cm}$.

The line intensity $I$ emitted by the plasma is sensitive to both the electron temperature $T_{e}$ and the local plasma density $n_{e}[11,12,13,14]$

$$
I=n_{0} \cdot F\left(n_{e}, T_{e}\right)
$$

Here $n_{0}$ is the particle density of the neutral gas and $F$ parametrizes the atomic physics processes involved in line emissions. A study on the exact dependency of $F$ on $n_{e}$ and $T_{e}$ revealed that $F \sim n_{e}^{\alpha} T_{e}^{\beta}$, where $\alpha=0.5-0.8$ and $\beta=0.3-1.4$ for values of $n_{e}$ and $T_{e}$ relevant to Alcator C-Mod [13].

We do not attempt to reconstruct the local particle density and temperature from GPI data and identify blob structures using the light emission intensity field.

In Fig. 2 we show the field-of-view of the camera overlaid with indicators of the magnetic geometry. At $R \simeq 89 \mathrm{~cm}$ the separatrix is denoted by the dashed line and at $R \simeq 91 \mathrm{~cm}$ the limiter shadow is denoted by the dashed-dotted line. The gap between these lines is the scrape-off layer, where the magnetic field lines intersect sheaths formed at the divertor targets. In this gap we define a triggering domain $\mathcal{T}$ of $10 \times 32$ pixels, denoted by the white rectangle in Fig. 2, and use it to detect blobs.

All intensity events exceeding $\zeta=1.5$ times the mean fluctuation in each point of the triggering domain,

$$
I\left(r_{i}, z_{i}, t\right)>\zeta \times \bar{I}\left(r_{i}, z_{i}\right) \forall\left(r_{i}, z_{i}\right) \in \mathcal{T}
$$

are recorded and sorted by amplitude in a list $L$. We assume all items in $L$ to be caused by blob events. A new list $L^{\prime}$ is constructed by iterating over L, starting with maximum amplitude. An item $L_{k}$ from $L$ is inserted into $L^{\prime}$ under the condition that no other item in $L^{\prime}$ is within 10 consecutive frames of $L_{k}$. Each element $L_{k}^{\prime}$ in $L^{\prime}$ contains the reference time, position and amplitude for a particular blob event. The chosen delay of 10 frames, 
corresponding to $25 \mu \mathrm{s}$, is motivated by the empirical observation that blobs usually occur more than $50 \mu \mathrm{s}$ apart. For each item $L_{k}^{\prime}$ in $L^{\prime}$, the blob in the reference frame is identified as the consecutive region around the reference peak with an intensity amplitude larger than $60 \%$ the intensity maximum. The blob event corresponding to $L_{k}^{\prime}$ is tracked by identifying regions of large intensity for up to 8 preceding and 8 succeeding frames, for a total of maximal 17 frames.

Starting from the reference frame of $L_{k}^{\prime}$, we identify all consecutive regions in the next frame where the intensity signal is at least $60 \%$ of the reference amplitude in $L_{k}^{\prime}$. If such a region exists it is taken to be the blob in the next frame, given that the conditions (i) the new regions intensity maximum is less than $7.5 \mathrm{~mm}$ away from the blobs local intensity maximum in the current frame and (ii) the new regions size is at least $10 \%$ of the current blobs size, are satisfied. If multiple regions satisfy this condition exist, we take the blob to be the region whose local intensity maximum is closest to the current blobs. Advancing to the next frame, this scheme is repeated until no regions satisfying (i) and (ii) can be found in the next frame.

For each blob event, we compute center-of-mass coordinates for the blob and center-ofmass velocities using a first order forward finite-difference scheme. If the center-of-mass coordinate of any blob event lies in the scrape-off-layer for 5 or fewer frames the event is discarded.

A blob events radial- and poloidal cross-field size is computed for each frame by fitting a gaussian function to its radial and poloidal cross-section at the local maximum of its intensity field. For each frame, we vary the initial fit interval of 8 pixels by \pm 2 and \pm 4 pixel and select the fit yielding the lowest mean-square error. The blobs radial and poloidal cross-field sizes $\ell_{\text {rad }}$ and $\ell_{\text {pol }}$ are then given by the respective fit parameter and stored together with the fit error for each frame. For a single blob event we define its mean cross-field sizes to be the weighted mean over the frames where the blobs mass center is in the SOL and the blobs velocity to be the mean over the frames, where the blobs mass center is in the SOL. 


\section{Results and discussions}

A scan of line-averaged density in ohmically heated, lower single-null plasmas in Alcator C-Mod is analyzed, shots $1120217-008,010,011,012,014,015,016,019,020$ and 020 are analyzed. For an on-axis magnetic field of $B_{\mathrm{T}}=4.0 \mathrm{~T}$ and a poloidal current of $I_{\mathrm{p}}=$ 0.6 MA, the line-averaged density $\bar{n}_{\mathrm{e}} / n_{G}$ is varied from 0.15 to 0.48 , where $n_{G}$ is the Greenwald density [15]. To increase the blob sample size for a given line-averaged density, we combine the blob events from shots with the same line-averaged density. In Fig. 3 we present the cross-field blob sizes and velocities obtained by the blob-tracking method discussed in the previous paragraph. For the plasma discharges, we detect typically between 66 and 350 blobs. Each data point presents the mean over all blobs detected in a single discharge. The radial blob velocities increase from $V_{\mathrm{rad}} \simeq 150 \mathrm{~m} \mathrm{~s}^{-1}$ for $\bar{n}_{\mathrm{e}} / n_{G} \simeq$ 0.15 to $V_{\mathrm{rad}} \simeq 450 \mathrm{~m} \mathrm{~s}^{-1}$ for $\bar{n}_{\mathrm{e}} / n_{G} \simeq 0.48$. The average vertical blob motion is in the negative Z-direction, the direction of the ion diamagnetic drift, with a mean velocity of $V_{\mathrm{pol}} \simeq-150 \mathrm{~m} \mathrm{~s}^{-1}$ for $\bar{n}_{\mathrm{e}} / n_{G}=0.15$. The range of observed poloidal blob velocities is larger than the range of radial blob velocities by a factor of $\sim 2$. For $\bar{n}_{\mathrm{e}} / n_{G}=0.48$, the observed mean poloidal blob velocity increases to $V_{\mathrm{pol}} \simeq-85 \mathrm{~m} \mathrm{~s}^{-1}$, again with a larger variation than the observed radial blob velocities.

We find that the blob's cross-field size remains roughly constant $\ell_{\text {rad }} \simeq \ell_{\text {pol }} \simeq 6.5 \mathrm{~mm}$ for all $\bar{n}_{\mathrm{e}}$. A weighted linear fit for the radial blob size yields $\ell_{\text {rad }}\left(\bar{n}_{\mathrm{e}} / n_{G}\right)=6.6 \mathrm{~mm}+1.4 \mathrm{~mm}$. $\bar{n}_{\mathrm{e}} / n_{G}$ and $\ell_{\mathrm{pol}}\left(\bar{n}_{\mathrm{e}} / n_{G}\right)=6.5 \mathrm{~mm}+1.03 \mathrm{~mm} \cdot \bar{n}_{\mathrm{e}} / n_{G}$ for the poloidal blob size.

We continue by comparing the observed blob statistics to results from sheath-connected blob theory. Estimating $T_{i}=T_{e}=10 \mathrm{eV}$ and $L_{\|}=5 \mathrm{~m}$, we evaluate $\ell_{*}=2.1 \mathrm{~mm}$ and $V_{*}=2.5 \mathrm{~km} \mathrm{~s}^{-1}$. In Fig. 4 we compare the observed blob velocities to the predicted radial blob velocities as a function of their poloidal cross-field size. All blob events from shots with equal line-averaged density are combined and each data point corresponds to an individual blob event.

Typical blob amplitudes in the scrape-off layer are of order unity, so $\triangle n / N \sim 1$. The curves for $\triangle n / N=0.1$ and 5 are used as an estimate of upper- and lower- bounds for blob velocities in the SOL. 
We find that in terms of dimensionless variables, the blob velocity increases from $V / V_{*}=$ $0.07 \pm 0.05$ for $\bar{n}_{\mathrm{e}} / n_{G}=0.15$ to $V / V_{*}=0.15 \pm 0.07$ for $\bar{n}_{\mathrm{e}} / n_{G}=0.48$, while the blob size remains constant at $\ell / \ell_{*} \simeq 3$. With increasing $\bar{n}_{\mathrm{e}} / n_{G}$, an increasing number of blob events systematically exceed the theoretically predicted radial blob velocity from sheathdissipated blob theory. This shows that local plasma parameters influence the radial propagation of blobs in a way that is not captured by simplified blob theory.

\section{Conclusion}

We have developed a blob-tracking algorithm that measures radial and poloidal blob crossfield sizes and their radial and poloidal velocity. This algorithm is used to study blob dynamics in the SOL for a scan in line-averaged density in Alcator C-Mod. We find that for large line-averaged plasma densities, the radial blob velocities systematically exceed predictions from blob theory for sheath-dissipated blobs. The size of the observed blobs varies little with discharge parameters and is well above the length on which one would expect the inertial velocity scaling to hold. Inertial velocity scaling overestimates the blob velocity at least by a factor of three. The radial velocities and sizes are consistent with results from other works which employ a correlation method and report $V_{\text {rad }} \simeq 300 \mathrm{~m} \mathrm{~s}^{-1}$ for $\bar{n}_{\mathrm{e}} / n_{G}=0.2$ which increases to $V_{\mathrm{rad}} \simeq 1600 \mathrm{~m} \mathrm{~s}^{-1}$ for $\bar{n}_{\mathrm{e}} / n_{G}=0.75[16,17]$. These results bolster the conjecture that local plasma parameters, as plasma collisionality and resistivity, as well as the parallel dynamics of the blob are important for determining the radial velocity of blobs in the scrape-off layer. 


\section{References}

[1] S. I. Krasheninnikov, Phys. Lett. A 283, 368 (2001).

[2] D. A. D'Ippolito, J. R. Myra and S. I. Krasheninnikov, Phys. Plasmas 9, 222 $(2002)$.

[3] D. A. D'Ippolito, J. R. Myra and S. J. Zweben, Phys. Plasmas 18, 060501 (2011).

[4] O. E. Garcia, N. H. Bian and W. Fundamenski, Phys. Plasmas 13, 082309 (2006).

[5] R. Kube and O. E. Garcia, Phys. Plasmas 18, 102314 (2011).

[6] C. Theiler, I. Furno, P. Ricci et al., Phys. Rev. Lett. 103, 065001 (2009).

[7] J. R. Myra, D. A. Russel and D. A. D'Ippolito, Phys. Plasmas 13, 112502 (2006).

[8] O. E. Garcia, J. Horacek, R. A. Pitts et al., Nucl. Fusion 47, 667-676 (2007).

[9] D. A. Russel, D. A. D’Ippolito, J. R. Myra et al., Phys. Rev. Lett. 93, 265001 (2004).

[10] B. LaBombard, R. L. Bovin, M. Greenwald et al., Phys. Plasmas 8, 2107 (2001).

[11] S. J. Zweben, D. P. Stotler, J. L. Terry et al., Phys. Plasmas 9, 1981 (2002).

[12] J. L. Terry, S. J. Zweben, K. Hallatschek et al., Phys. Plasmas 10, 1739 (2003)

[13] D. P. Stotler, B. LaBombard, J. L. Terry et al., J. Nucl. Mater. 313-316, 10661070 (2003).

[14] J. L. Terry, N. P. Basse, I. Cziegler et al., Nucl. Fusion 45, 1321-1327 (2005).

[15] M. Greenwald, Plasma Phys. Control. Fusion 44, R27 (2002).

[16] M. Agostini, J. L. Terry, P. Scarin et al., Nucl. Fusion 51, 053020 (2011).

[17] S. J. Zweben, J. L. Terry, M. Agostinit et al., Plasma Phys. Controlled Fusion 54, 025008 (2012). 


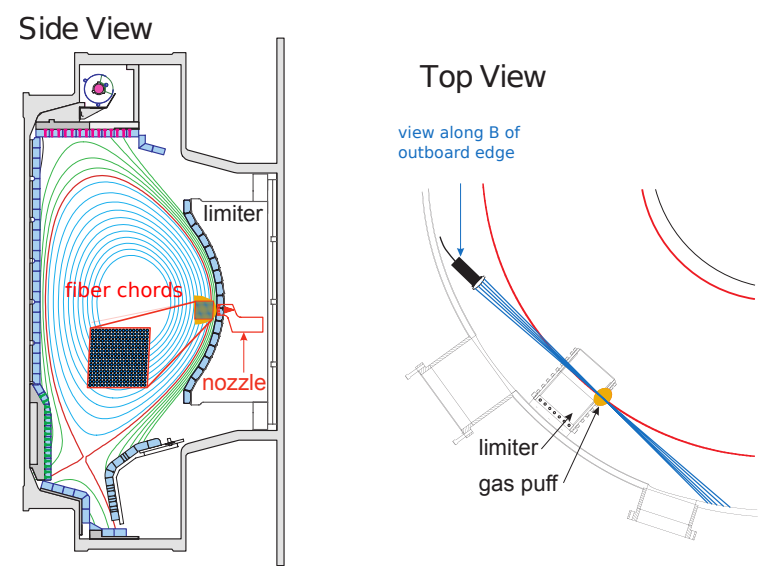

Figure 1: Schematics of the GPI diagnostic installed in Alcator C-Mod, side- and topview. The fiber chord view is aligned along the magnetic field.

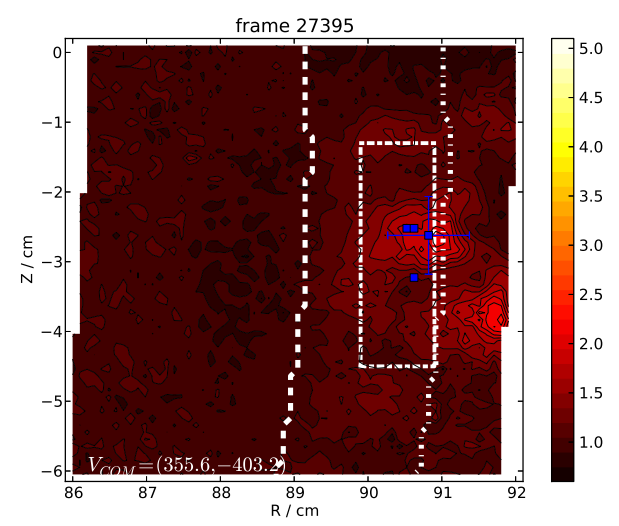

Figure 2: Field of view of the fast camera. Overlaid are the separatrix (dashed line) at $R \simeq 89 \mathrm{~cm}$, the wall shadow at (dashed-dotted line) at $R \simeq 91 \mathrm{~cm}$, and the triggering domain (dashed-dotted rectangle). The blue square marks are the center-of-mass of the blob structure for all previous frames in the blob trail and the blue error bars denote the blob size for the current frame.
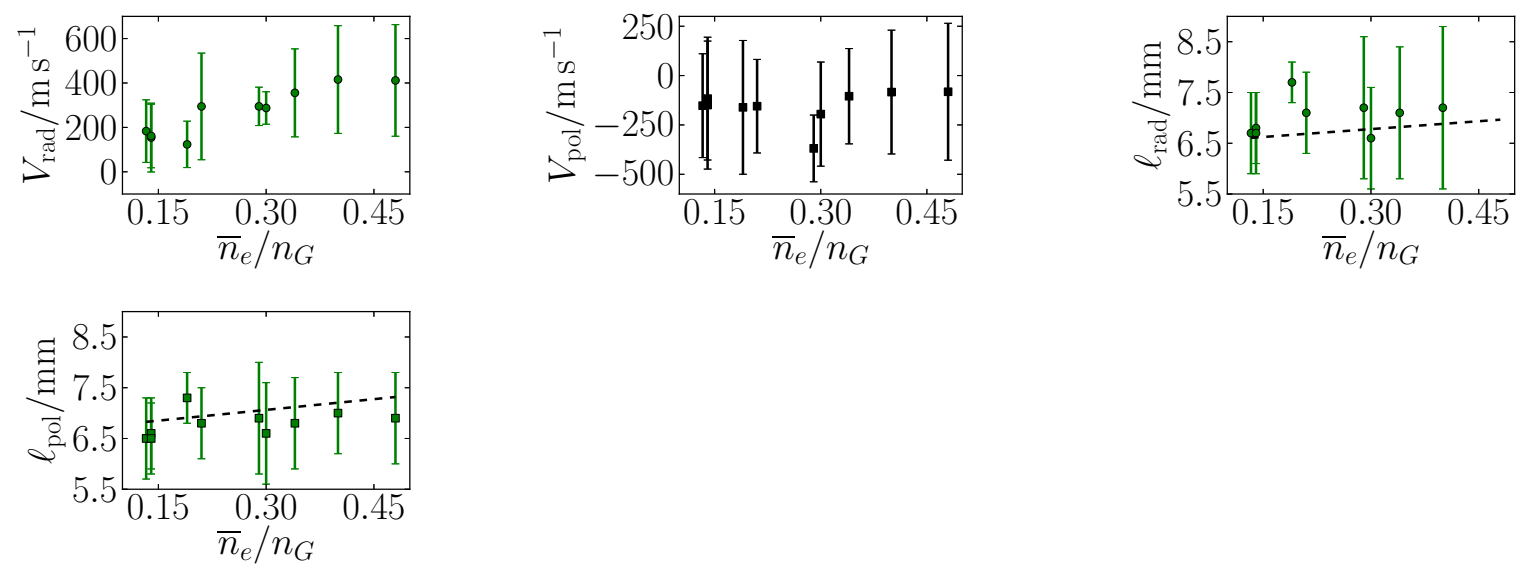

Figure 3: Observed blob center-of-mass velocities and cross-field sizes as a function of discharge plasma density $\bar{n}_{\mathrm{e}} / n_{G}$. The black lines are weighted linear fits on the blob size and their parameters given in the text. 


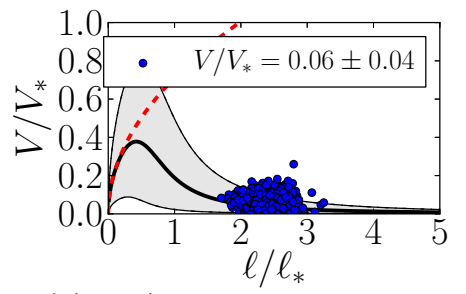

(a) $\bar{n}_{\mathrm{e}} / n_{G}=0.15,741$ events

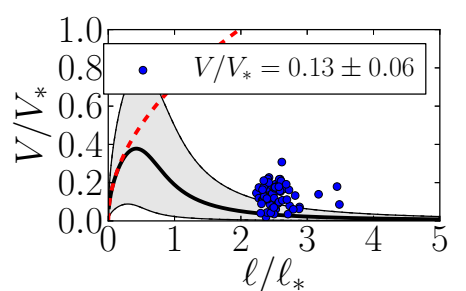

(d) $\bar{n}_{\mathrm{e}} / n_{G}=0.48,66$ events

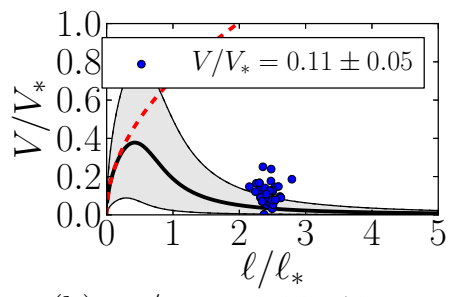

(b) $\bar{n}_{\mathrm{e}} / n_{G}=0.30,41$ events

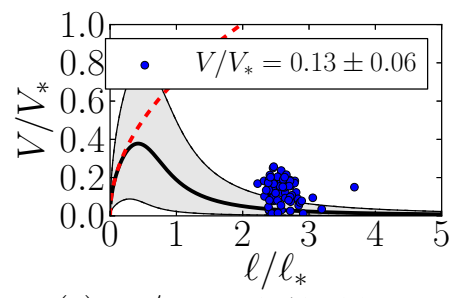

(c) $\bar{n}_{\mathrm{e}} / n_{G}=0.40,81$ events

Figure 4: Observed radial blob velocities as a function of poloidal cross-section. Overplotted are predicted radial blob velocities as a function of cross-field size for relative blob amplitudes $\triangle n / N=1$ (full line), curves for $\Delta n / N=0.1,5$ estimate an error interval (colored area). The dashed line is the theoretical maximum velocity for blobs given by the inertial velocity scaling. 